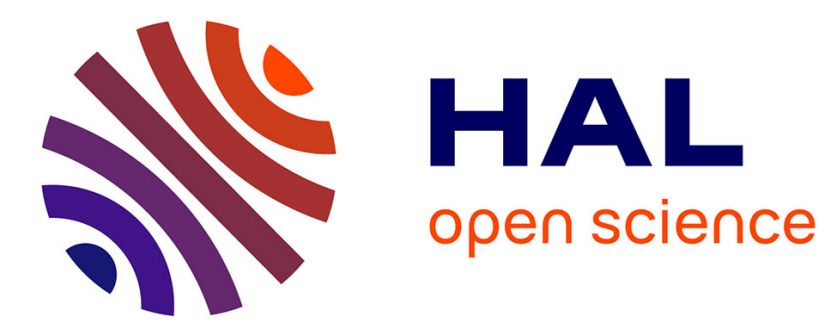

\title{
Experimental study of a free and forced swirling jet
}

François Gallaire, S. Rott, Jean-Marc Chomaz

\section{To cite this version:}

François Gallaire, S. Rott, Jean-Marc Chomaz. Experimental study of a free and forced swirling jet. Physics of Fluids, 2004, 16 (8), pp.2907-2917. 10.1063/1.1758171 . hal-01024955

\section{HAL Id: hal-01024955 \\ https://hal-polytechnique.archives-ouvertes.fr/hal-01024955}

Submitted on 29 Jul 2014

HAL is a multi-disciplinary open access archive for the deposit and dissemination of scientific research documents, whether they are published or not. The documents may come from teaching and research institutions in France or abroad, or from public or private research centers.
L'archive ouverte pluridisciplinaire HAL, est destinée au dépôt et à la diffusion de documents scientifiques de niveau recherche, publiés ou non, émanant des établissements d'enseignement et de recherche français ou étrangers, des laboratoires publics ou privés. 


\section{AIP | Physics of Fluids}

\section{Experimental study of a free and forced swirling jet}

F. Gallaire, S. Rott, and J.-M. Chomaz

Citation: Physics of Fluids (1994-present) 16, 2907 (2004); doi: 10.1063/1.1758171

View online: http://dx.doi.org/10.1063/1.1758171

View Table of Contents: http://scitation.aip.org/content/aip/journal/pof2/16/8?ver=pdfcov

Published by the AIP Publishing

\section{Articles you may be interested in}

Large eddy simulation of flow development and noise generation of free and swirling jets

Phys. Fluids 25, 126103 (2013); 10.1063/1.4833215

Global modes in a swirling jet undergoing vortex breakdown

Phys. Fluids 23, 091102 (2011); 10.1063/1.3640007

Peculiar phenomenon of micro-free-jet flow

Phys. Fluids 21, 092001 (2009); 10.1063/1.3224012

Confined swirling jet impingement on a flat plate at moderate Reynolds numbers

Phys. Fluids 21, 013601 (2009); 10.1063/1.3063111

A numerical study of dynamics of a temporally evolving swirling jet

Phys. Fluids 13, 951 (2001); 10.1063/1.1350876

\section{AlP hameded}

Journal of Applied Physics is pleased to announce André Anders as its new Editor-in-Chief 


\title{
Experimental study of a free and forced swirling jet
}

\author{
F. Gallaire, ${ }^{\text {a) }}$ S. Rott, and J.-M. Chomaz \\ Laboratoire d'Hydrodynamique (LadHyX), CNRS, École Polytechnique, 91128 Palaiseau Cedex, France
}

(Received 4 February 2004; accepted 3 April 2004; published online 1 July 2004)

\begin{abstract}
The present study concerns the response of a swirling jet to various azimuthal modes and frequencies forced at the nozzle exit. The different unforced dynamical states are first described as a function of the swirl setting, determined from measured velocity fields in the longitudinal plane using particle image velocimetry. A second experimental technique, based on laser induced fluorescence, is described, which is more suited to the description of the low-amplitude response of the jet to the forcing. It is shown that the receptivity of the jet is very poor when the forcing is set to the naturally prevailing azimuthal mode $(m=2)$ and frequency. In contrast, a strong response is observed for both co-rotating and counter-rotating forced azimuthal modes $(m= \pm 2, m= \pm 3)$ for frequencies about one order of magnitude larger than the frequency prevailing in the absence of forcing. Finally, the present actuator is seen to be ineffective in preventing the appearance of vortex breakdown itself. (C) 2004 American Institute of Physics. [DOI: 10.1063/1.1758171]
\end{abstract}

\section{INTRODUCTION}

Vortex breakdown is a widespread phenomenon that is encountered in aeronautical industrial applications as well as atmospheric conditions. It is known to alter considerably the flow characteristics resulting in either desirable features such as flame-holders in combustion devices or undesirable consequences, for instance on delta-wings as reviewed in Ref. 1 or 2. In the latter flow-configuration, the high technological issue at stake has motivated researchers to develop control strategies (see Ref. 3 for a review). But only a few studies have dealt with the control of the more academic experimental setups in tubes and free swirling jets. These flowconfigurations have enabled the discovery of a large variety of flow-states ${ }^{4}$ where helical and axisymmetric breakdowns are in competition. Since there is no general agreement on whether vortex breakdown is basically an axisymmetric process or how helical modes contribute in the breakdown process, we examine the response of a swirling jet to helical perturbations in both the prebreakdown and the breakdown regime as defined in Ref. 5.

A recent study by Panda and McLaughlin ${ }^{6}$ was aimed at determining the influence of swirl on the amplification of unstable modes in a free swirling air jet at Reynolds numbers above $R e=20000$ in the regime preceding vortex breakdown. In the unforced regime, these authors established the absence of any streamwise growth of the perturbations. An acoustic forcing device was then arranged circumferentially around the jet axis, consisting of four large loudspeakers. Various phase combinations between speakers were possible and different instability modes could be excited, developing into organized structures consisting of both axisymmetric $(m=0)$ and bending modes $(|m|=1)$.

The present study, after presenting some new measure-

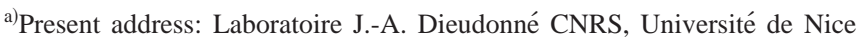
Sophia Antipolis, Parc Valrose, F-06108 Nice Cedex 02, France. Electronic mail: francois@ladhyx.polytechnique.fr
}

ments of the free swirling jet experiment developed by Billant, Chomaz, and Huerre ${ }^{7}$ at LadHyX, describes the response of the swirling jet to various time-dependent azimuthal perturbations generated at the nozzle exit. In the prebreakdown stage, the flow is shown to respond most intensively at substantially higher frequencies than that of the natural mode in the absence of forcing. In contrast, vortex breakdown occurs at the same value of the swirl parameter, no matter what frequencies or azimuthal modes are applied to force the flow.

The paper is organized as follows. Section II briefly presents the experimental setup and forcing device, while the diagnostic tools are described in Sec. III. Section IV is devoted to a description of the flow states in absence of forcing whereas the forced experiments are analyzed in Sec. V, with a final discussion concluding the paper.

\section{EXPERIMENTAL SETUP}

\section{A. Background}

The present experimental study makes use of the same experimental apparatus as the study of Billant et al. ${ }^{7}$ and Loiseleux and Chomaz ${ }^{5}$ and we therefore briefly review their main results. Billant et al. ${ }^{7}$ thoroughly measured the axial and azimuthal velocity profiles at the nozzle exit using the laser Doppler velocimetry (LDV) technique. The azimuthal profile was characterized by a core in nearly solid body rotation with a rapid decrease to a zero azimuthal velocity associated to an outer annulus of negative vorticity. Such a shielded vortex is usually referred to as an isolated or screened vortex and is known to promote both the axisymmetric centrifugal instability as well as the destabilization of azimuthal modes resulting from the Kelvin-Helmholtz instability associated to the azimuthal shear, i.e., to the rapid variation of the azimuthal velocity in the radial direction (see Ref. 8 for a review). This contrasts with the celebrated 


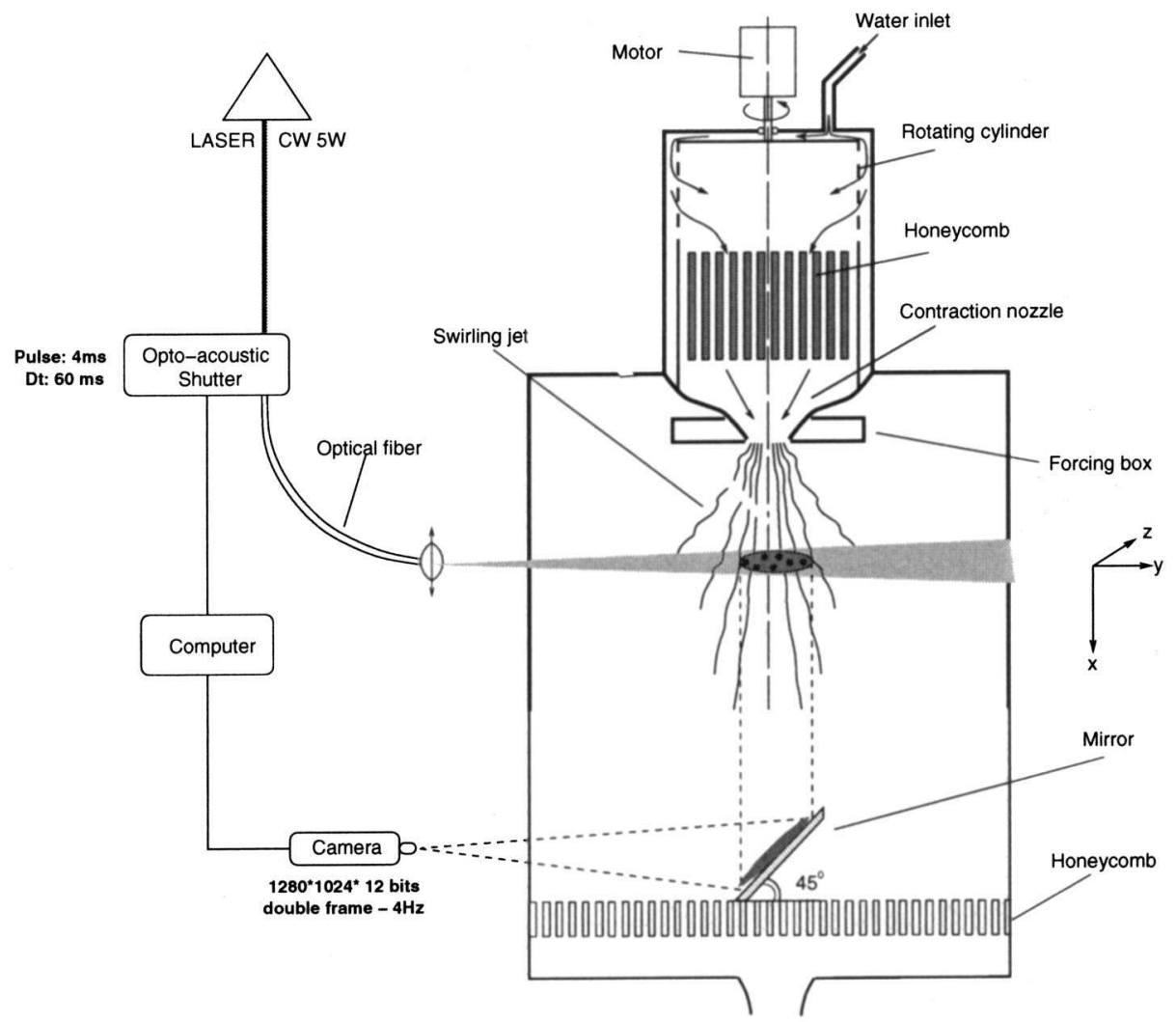

FIG. 1. Experimental setup and PIV in the horizontal plane.

Lamb-Oseen vortex with single-signed axial vorticity. Furthermore, the axial velocity profile was seen to present a mild axial shear and a velocity overshoot in the vicinity of the axis of the jet which increases with the swirl. Billant et $a .^{7}$ argued that this phenomenon is a consequence of the nonlinear interaction between the axial and azimuthal velocity components in the contraction nozzle.

Flow-visualizations were carried out using longitudinal and cross-sectional laser induced fluorescence (LIF) techniques. This experimental technique reveals most of the evolution of the flow as the swirl parameter is increased. In the Reynolds number range $300 \leqslant \mathrm{Re} \leqslant 1200$, Billant et al. ${ }^{7} \mathrm{dem}-$ onstrated that the swirl number, as defined in Eq. (1), was indeed an adequate control parameter for the determination of the onset of vortex breakdown. Vortex breakdown occurs for a swirl setting of $S=S_{c} \sim 1.3$, for all Reynolds numbers considered. Four distinct types of vortex breakdown were identified. In addition to the steady and precessing bubble vortex breakdown types, evidence was given of a new type of vortex breakdown, the cone (both in its steady and precessing form). The LDV technique was used to document the axial and radial velocity field in the case of a bubble at $\operatorname{Re} \sim 670$.

Striking helical structures were observed for parameter settings prior to vortex breakdown, independent of the Reynolds number. Helical structures of wavenumber $m=2$ (trident) and $m=3$ (celtic cross) were observed. They were observed to rotate at very low or nearly vanishing frequencies and small axial wavenumbers corresponding to a large pitch of the helix. The sense of their winding was the same as that of the rotation of the jet.

In a related study making use of the same experimental device, Loiseleux and Chomaz ${ }^{5}$ focused on the early development of the instabilities at $\mathrm{Re}=1490$, immediately at the nozzle exit. Adequate image processing (as described in more detail in the next section) enabled them to distinguish between three flow regimes. The first regime $S \in[0 ; 0.6]$ is characterized by the axisymmetric Kelvin-Helmholtz instability very reminiscent of the nonswirling round jet. The roll-up of the Kelvin-Helmholtz rollers is precisely the feature which prevents quantitative measurements based on the LIF visualizations further away from the nozzle exit, since in that case, the radius of the jet cannot be defined unambiguously. The second regime $S \in[0.6 ; 1]$ is characterized both by the axisymmetric Kelvin-Helmholtz instability but also by an $m=+2$ co-rotating helical instability. The last regime which precedes breakdown $S \in[1 ; 1.3]$ is very unstationary and irregular. Only Fourier transforms revealed the presence of an $m=-1$ counter-rotating structure.

\section{B. Experimental setup}

The experimental setup used in this study has been described in Ref. 7 and is shown schematically in Fig. 1.

The main characteristic of the apparatus resides in the fact that rotation and advection are generated independently. This leads naturally to the definition of two independent non- 


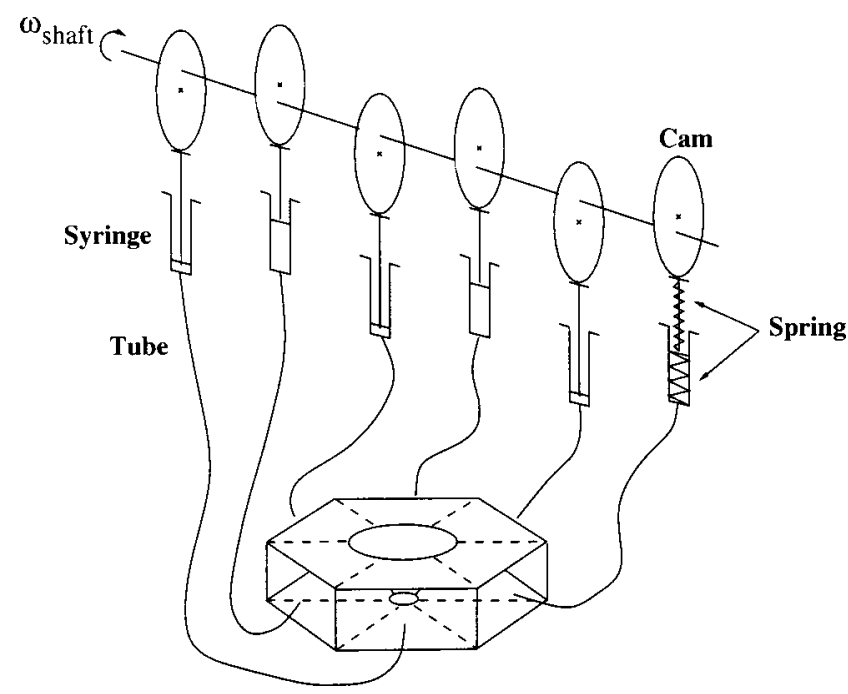

FIG. 2. Forcing device.

dimensional parameters. The swirl number is defined according to

$$
S=\frac{2 U_{\theta}(r=R / 2)}{U_{x}(r=0)} .
$$

The swirl number $S$ characterizes the intensity of the rotation with respect to the axial advection. The Reynolds number, characterizing the advection, is based on the maximum velocity of the jet in the absence of rotation and on the diameter of the jet according to

$$
\operatorname{Re}=\frac{2 U_{x}(r=0, S=0) R}{\nu} .
$$

The forcing device is presented in Fig. 2. As in Ref. 9 it consists of a forcing box divided into six cells of the same size filled with water. Each cell has a small aperture of size $2 \mathrm{~mm}$ which is near the nozzle and through which water is pumped in or out. Each cell is connected to a pulsing device by a tube filled with water. Different pulsing devices were used. Loudspeakers have the advantage of a precise determination of the forcing amplitude and offer a total freedom in the choice of the actuator signal. However, they work only for frequencies higher than $5 \mathrm{~Hz}$.

For forcing frequencies less than $5 \mathrm{~Hz}$, we developed a cam shaft controlling syringes (Fig. 2); this has the restriction of imposing periodic signals. The amplitude of the signal can be varied by changing the interaxis distance, within a limited range of amplitudes.

Because of the finite number of forcing points, these forcing devices can only excite modes $|m| \leqslant 6$. Furthermore, whenever a mode $m$ is forced, the mode $m-6$ is also forced. The modes are thereby forced in pairs $(0),(+1,-5),(+2$, $-4),(+3,-3),(+4,-2)$ and $(+5,-1)$.

For either a given amplitude of the loudspeaker membrane motion or a given value of the cam shaft interaxis length, the forcing device provokes a forcing at constant volume for any value of the frequency $\omega$. As a consequence, the side-jet velocity increases linearly with $\omega$. We have therefore verified that the forcing velocity never exceeds $1 \%$ of the mean axial velocity.

\section{DIAGNOSTIC TOOLS}

Two experimental techniques are used in the present study. Particle image velocimetry (PIV) has been used to describe the dynamical flow-states. Laser induced fluorescence (LIF) in the cross-sectional plane immediately at the nozzle exit together with sequential image processing has also been used in order to characterize the response of the swirling jet to a time-periodic forcing. The same continuous $5 \mathrm{~W}$ argon laser is used for the global PIV measurements (together with an opto-acoustic shutter) and for the LIF cross-sectional images.

\section{A. PIV}

Since the velocity fields are three-dimensional, PIV measurements require precise parameter settings which are briefly described herein. We use the PIV FlowMaster3 system manufactured by LaVision which consists of an ultrasensitive digital camera by Kodak, a hardware synchronization unit and a software for the correlation algorithm. In addition, we use an opto-acoustic shutter to produce laserpulses from a continuous laser.

The PIV works in double-frame/double-exposure mode in the sense that each flow evaluation results from the crosscorrelation of two physical frames exposed at two different laser flashes. The resolution of the CCD camera is 1280 $\times 1084$ with a sensitivity of 12 bits (i.e., 4096 light intensity levels). The acquisition frequency of the camera is $4 \mathrm{~Hz}$, i.e., the camera shoots a pair of frames four times a second. This acquisition frequency is too low for dynamical studies and PIV is therefore devoted in our experiment to the characterization of the flow-states.

The two frames are separated by the time interval $\delta t=60 \mathrm{~ms}$, which is one of the most important control parameters. This time interval is chosen so as to minimize the number of in-plane and cross-plane lost particles but must also remain greater than the exposure time. Since our laser is only $5 \mathrm{~W}$, the exposure time is chosen relatively long $t_{\text {exp }}=4 \mathrm{~ms}$, in order to illuminate the camera sufficiently so as to make the most out of the high sensitivity of the camera. This exposure time is a compromise between having a neat image with steady particles and no streaks and having enough light. In order to obtain as much light as possible we use a multimode fiber. At the end of the fiber, a set of cylindrical lenses produces a laser sheet of thickness $\delta z$. The selection of $\delta z$ is a trade-off: it seeks simultaneously to maximize the number of illuminated particles (proportional to $\delta z$ ), to maximize the intensity received and reflected by each particle (proportional to $1 / \delta z$ ) while also to minimize the number of lost out-of-plane particles (proportional to $1 / \delta z)$. The density of particles is then chosen so as to have at least three particles in the interrogation window. The particles are chosen to be as bright as possible and to have an approximate size of $2 \times 2$ pixels, thereby diminishing the peak-locking effect. ${ }^{10}$ We use $\mathrm{TiO}_{2}$ particles of mean diameter 200 microns. 


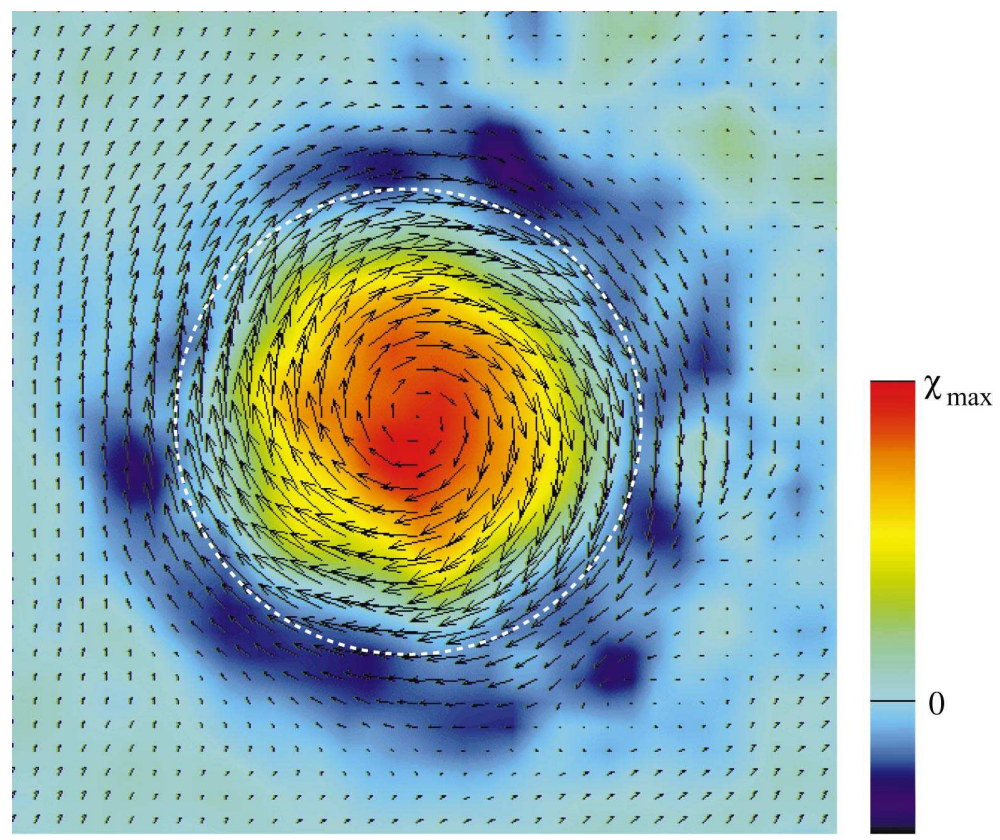

FIG. 3. (Color) Velocity and color coded vorticity fields in a transverse plane measured with PIV at $S=0.8$, $\operatorname{Re}=900$, and $X=0.6$.
The cross-correlations are evaluated with standard FFTs. The flow fields are calculated by means of an adaptative multipass algorithm which gradually decreases the size of the interrogation windows to estimate the shift from the results at the previous steps for better window-matching. The initial interrogation windows are $128 \times 128$ and the final ones are $32 \times 32$ with an overlap of $50 \%$. The final algorithm used consists of a sub-pixel displacement evaluation through a three point Gaussian cross-correlation peak fitting.

For velocity measurements in the streamwise plane the camera and laser sheet are easily aligned with a symmetry plane of the swirling jet, whereas for measurements in crosssectional planes, we use a mirror leaning at 45 degrees which avoids parallax errors which would have occurred if the camera lens had been tilted with respect to the laser sheet. This method nevertheless presents several drawbacks: the loss in luminosity during the optical path in the seeded water, as well as the necessity to focus the camera and to calibrate the image each time the cross-sectional streamwise location $X=x / D$ is varied. Figure 3 depicts a typical velocity field in the transverse plane just at the nozzle exit $X=0.6$ for $\mathrm{Re}=900$ and $S=0.8$. In addition, the corresponding axial vorticity component is plotted with pseudocolor on the same image. The vorticity calculated via discrete finite difference formulas complemented by a Gaussian filter shows the existence of an annulus of opposite vorticity screening the vortex (Fig. 3).

\section{B. Spatio-temporal diagrams}

Although LIF is generally used to provide qualitative insights, Loiseleux and Chomaz ${ }^{5,9}$ have also demonstrated how to extract quantitative results from LIF movies, using the same experimental apparatus. We take advantage of the fact that, if the tracer is homogeneously mixed in the honeycomb before entering the contraction, the dye delineates a vorticity surface at the nozzle exit. If viscous effects are neglected, the Kelvin theorem specifies that a vorticity surface evolves as a material surface. The dye interface stays therefore a vorticity surface until far downstream where corrugation of the surface is strong enough for the diffusion of vorticity to be significant. Following the dye perturbations at shorter timescales than vorticity diffusion provides information about the vorticity sheet deformations which characterize the intensity and development of flow-perturbations. This contrasts to flow visualizations based on a single filament of dye at the swirling jet centerline which is not a particular vorticity line (except if the centering is perfect).

Figure 4 displays a cross-plane LIF cut at a downstream station $X=1$ in the case of $S=0.86$ and a forcing of the $m=-2$ mode at nondimensional forcing frequency $\omega_{f}=7$ (see the next section). In this case, the double helix disturbance (black arrow) rotates in time in the direction opposite to that of the underlying swirling jet (white arrow). The radial position of the dye interface is first extracted out of a movie as a function of the cylindrical angle $\alpha$ and time $t$ via image-processing built into the NIH Image software. In order to avoid roll-up of the dye and to guarantee a unique defini-

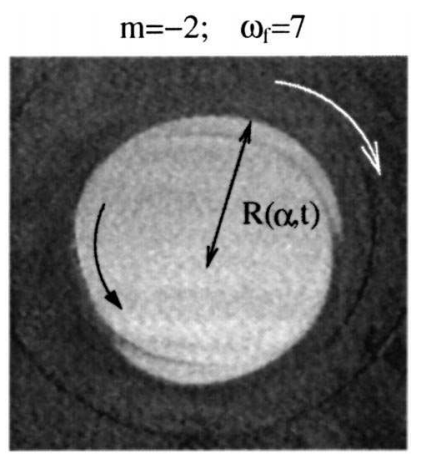

FIG. 4. Transverse cut produced by LIF; $\operatorname{Re}=1490, S=0.84, X=1$; $m=-2$ forcing at $\omega_{f}=7$. The dashed line corresponds to the nozzle exit radius. 


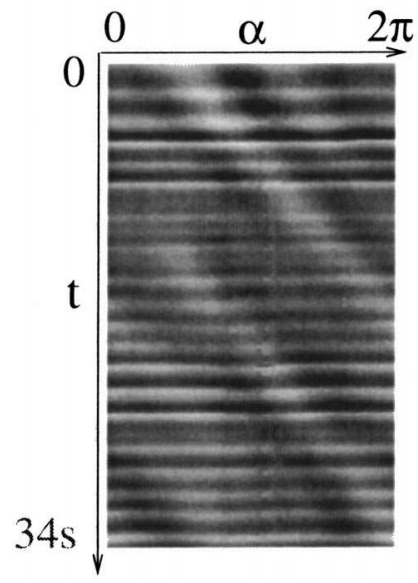

FIG. 5. Typical spatio-temporal diagram. The radius is represented as a gray level in the $\alpha-t$ spatio-temporal plane.

tion of the radius function $r(\alpha, t)$, the position $X$ of the laser sheet is kept close enough to the nozzle exit, resulting in general in values of $X$ less than 1 . Typical spatio-temporal diagrams are then obtained as shown in Fig. 5 for the unforced case at $S=0.86$, where the gray-level represents the radial deformation of the jet $r(\alpha, t)$ as a function of the angle $\alpha$ and time $t$.

On such a spatio-temporal diagram, the KelvinHelmholtz jet instability corresponding to axisymmetric modes $(m=0)$ is visible as the horizontal stripes. Azimuthal waves or instabilities $(m \neq 0)$ are readily identified by oblique stripes. This enables a precise determination of the azimuthal wavenumber $m$ as well as the phase-velocity $v_{\phi}$. When the swirl number is in the second regime $0.6<S<1$ defined by Loiseleux and Chomaz, ${ }^{5}$ two or three oblique stripes appear in addition to the characteristic horizontal stripes corresponding to the development of $m=2$ or $m=3$ on top of $m=0$ modes. The phase velocity of these $m=2$ or $m=3$ mode is lower than of the rotation rate of the vortex (about $\frac{1}{10}$ ) and proportional to the swirl number. Gallaire and Chomaz $^{11}$ have recently proposed a theoretical explanation to the double-helix mode selection in the second regime. The double-helix can be understood as a nonlinear self-sustained global mode relying on the absolutely unstable nature of the flow at the nozzle exit.

\section{FLOW STATES IN ABSENCE OF FORCING}

\section{A. Weakly rotating regime}

Figure 6(a) represents the measured meridional velocity of the nonswirling jet which is representative of the first regime $0 \leqslant S \leqslant 0.6$. As for nonrotating jets, the flow is dominated by the development of axisymmetric KelvinHelmholtz rings corresponding to the waviness of the streamlines and of the axial velocity contours. The corresponding transverse vorticity field $\eta$ is shown in Fig. 6(d) and demonstrates the rolling up of vorticity associated to the formation of Kelvin-Helmholtz rings. Throughout Figs. 6(a)-6(c), the color code associated to the axial velocity intensity is depicted in Fig. 6(a) and is rescaled for each sub- figure respectively, so as to cover the full range $0 \leqslant\left|U_{x}\right| \leqslant\left|U_{x}\right|^{\max }$ where $\left|U_{x}\right|^{\max }=\max \left(\left|U_{x}\right|\right)$ is the maximum absolute value of the axial velocity over the whole corresponding field. Along the same lines, throughout Figs. 6(d)6(f), the color code associated to the azimuthal vorticity is depicted in Fig. 6(d) and is rescaled for each subfigure respectively, so as to cover the full range $-\eta_{\max } \leqslant \eta \leqslant \eta_{\max }$, where $\eta_{\max }=\max (|\eta|)$ is the maximum absolute value of the vorticity over the shown field.

\section{B. Trident state}

The second regime $0.6 \leqslant S \leqslant 1$ is characterized by the development of a double spiral mode that appears on a meridional LIF image as a trident [see Fig. 7(a)], since the laser cuts the middle of the spiral and both arms that develop on the side. The velocity distribution obtained from PIV images shows similar features as seen in Fig. 6(b) obtained for $S=1.35$ and $\mathrm{Re}=900$. The high axial velocity region appears split in three as a result of the development of the double spiral. The perturbation corresponding to the remaining $m=0$ modes produces roll up of vorticity [as seen in Fig. 6(e)] in each of the arms. This state is not a breakdown state, since no stagnation point is present!

\section{Breakdown cone}

In Fig. 6(c), the transverse velocity field is represented for a swirl number of $S=1.35$ and Reynolds number of $\operatorname{Re}=900$. This velocity field elucidates the structure of the conical breakdown in complete agreement with the LIF visualizations of Billant et l. $^{7}$ reproduced in Fig. 7(b). What was assumed to be a stagnation point is effectively seen to stagnate. Further downstream in the cone, the velocity field is remarkably concentrated into a thin layer. The angle of the cone here is approximately $100^{\circ}$, in good agreement with the results of Billant et al. ${ }^{7}$

Still more insight can be gained while computing the vorticity field in the $z$-direction. Results are depicted in Fig. 6(f) for the same parameter settings as in Fig. 6(c). Positive vorticity values are depicted in red, negative in blue, whilst the hue (ranging form light to dark) reveals the absolute value of the vorticity. This figure should not be misinterpreted, since it represents the $z$-vorticity in a full meridional section; only the right part corresponds to the azimuthal velocity field for positive radii $r$ (at an azimuthal angle of 0 ) whereas the left part corresponds to the azimuthal velocity field for negative radii $r$ (and therefore to the opposite of the vorticity at the azimuthal angle of $180^{\circ}$ ). The axisymmetric structure of the cone suggested by Billant et al. ${ }^{7}$ is demonstrated since the left part of the velocity field is axially related by reflection to the right part. In the following, we comment on the right part of Fig. 6(f) corresponding to the usual convention of positive $r$. Upstream from breakdown, just at the nozzle exit, the vorticity is positive and concentrated in the jet shear layer.

The most striking fact is that vortex breakdown is also accompanied by substantial generation of negative azimuthal vorticity. There is indeed a mechanistic explanation of vortex 

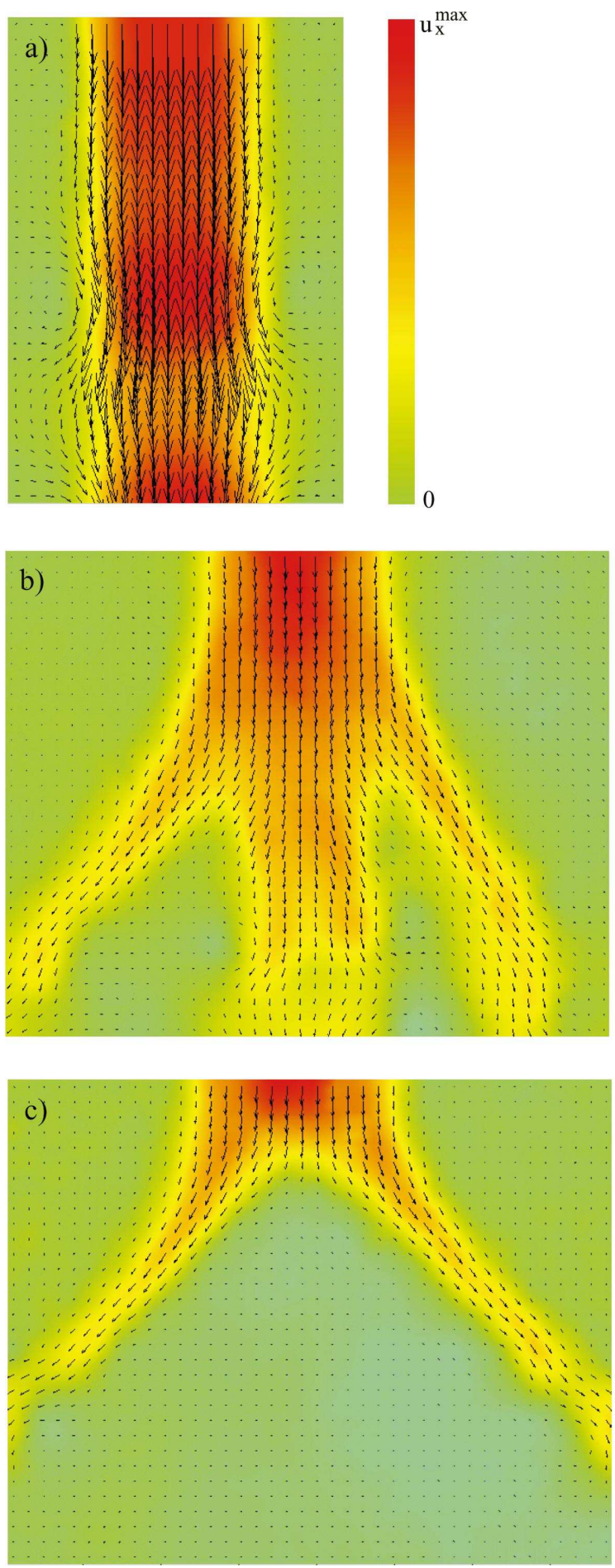
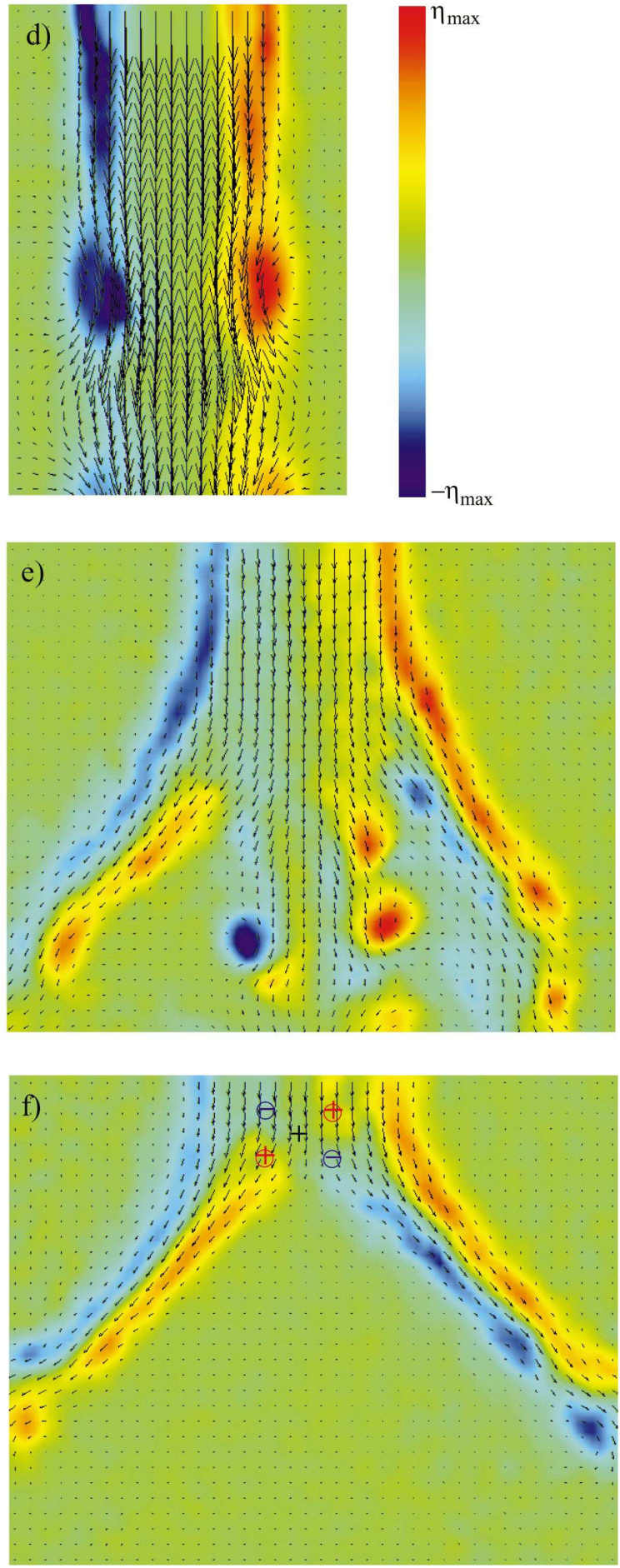

FIG. 6. (Color) Longitudinal velocity field and associated intensity of the axial component of velocity [(a)-(c)] and transverse $z$-vorticity component $\eta$ $[(\mathrm{d})-(\mathrm{f})]$ in the three regimes. (a) and (d) Regime I: longitudinal velocity field and $z$-vorticity component in a streamwise plane measured with PIV; $S=0$; $\mathrm{Re}=1200$. (b) and (e) Regime II: longitudinal velocity field and $z$-vorticity component in a streamwise plane measured with PIV; $S=0.8 ; \operatorname{Re}=900$. (c) and (f) Regime III (cone type breakdown): longitudinal velocity field and $z$-vorticity component in a streamwise plane measured with PIV; $S=1.35 ; \mathrm{Re}=900$. Note that the velocity scale varies from one subfigure to the other. Furthermore, the color code represented in (a) [respectively in (d)] is specific to each subfigure and scales from 0 to $U_{x}^{\max }$ (respectively from $-\eta_{\max }$ to $\eta_{\max }$ ).

breakdown proposed by Brown and Lopez, ${ }^{12}$ Darmofal $^{13}$ and Althaus et al., ${ }^{1}$ according to which vortex breakdown is nothing more than the end result of the tilting of axial vorticity due to the swirling motion into negative azimuthal vorticity. Furthermore, according to this line of thought, the appearance of negative azimuthal vorticity is a prerequisite for vortex breakdown, the characteristic stagnation point appearing in the flow only when a sufficient amount of negative vorticity has been produced. The appearance of negative azimuthal vorticity starts a positive feedback mechanism, which accelerates its production, finally leading to vortex breakdown. This scenario can be in particular identified in the 

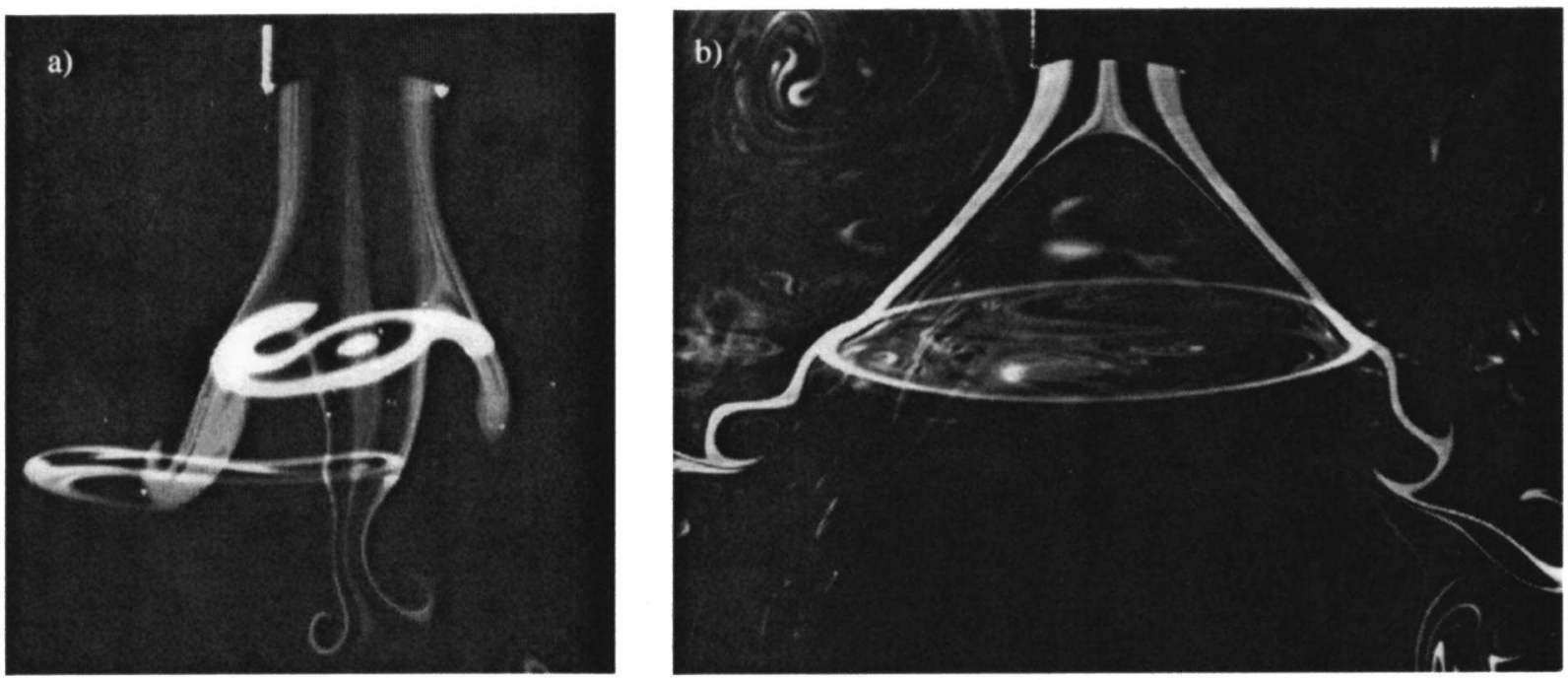

FIG. 7. (a) Trident state observed by Billant et al. (Ref. 7) at $S=1.41$ and $\operatorname{Re}=606$. (b) Cone type breakdown state observed by Billant $e t$ al. (Ref. 7) at $S=1.37$ and $\operatorname{Re}=606$.

streamwise development of the breakdown flow. There exists another particular point on the axis, upstream of the stagnation point, which might elsewhere or even better characterize the appearance of vortex breakdown. This point is denoted by a $X$ in Fig. 6(f). Upstream of this point, the azimuthal vorticity is positive for small values of the radius whereas downstream of this point the azimuthal vorticity is negative in the vicinity of the axis. In other words, the production and distribution of negative vorticity, when sufficient, is able to change the sign of the azimuthal vorticity near the axis. Rockwell, Ozgoren, and Sahin ${ }^{14}$ suggested that vortex breakdown might be more easily diagnosed by monitoring such points of sign change of vorticity. It was argued that, in contrast to the stagnation point, which is known to become unsteady as a consequence of the helical instabilities of the bubble itself, ${ }^{15,16}$ the point of sign change of vorticity might remain steadier and be therefore easier to identify.

\section{FORCED EXPERIMENTS}

In spatially developing flow, time-dependent forcing (referred to as the spatial instability approach) is the preferred experimental methodology, since it provides estimates of spatial growth rates. Our forcing device, in contrast to the corrugated nozzle used by Lasheras, Lecuona, and Rodriguez ${ }^{17}$ in their study of pure jets, can prescribe both the azimuthal wavenumber and frequency. In the present study, the spatial growth rate of the instabilities is, however, not accessible since the saturation of the rings restricts present measurements to $X<1$ for forcing frequencies such that the response of the swirling jet is large. On the other hand, the setup does not allow observation for $X$ closer to the nozzle exit than $X=0.6$. Use of the PIV technique is even more restricted to large perturbation amplitudes and therefore would not give access either to spatial growth rates. We consider instead the amplitude at a given streamwise coordinate $X=0.6$ as a function of the forcing frequency and azimuthal mode number. Although the natural structures prevailing in the unforced flow are co-rotating, we consider the response to co-rotating $(m>0)$ and counter-rotating $(m<0)$ perturbations of azimuthal wavenumbers which correspond to the observed modes in the second regime, i.e., double helices $(|m|=2)$ and celtic crosses $(|m|=3)$. It is emphasized that throughout the study the sign of $m$ refers to the propagation in time of the azimuthal disturbance in a cross-plane, in contrast with theoretical studies where it refers to the sign of winding in space of the perturbations. The Reynolds number is kept constant and equal to $\mathrm{Re}=1490$ and the swirl number to $S=0.84$, a swirl value of the second regime identified by Loiseleux and Chomaz. ${ }^{5}$

The forcing frequency $\omega_{f}$ is nondimensionalized with the natural prevailing frequency $\omega_{\text {nat }}=2 v_{\phi_{\text {nat }}}=50$ $\times 10^{-2} \mathrm{rad} \cdot \mathrm{s}^{-1}$ as estimated by Loiseleux and Chomaz ${ }^{5}$ or measured on the spatio-temporal diagram from Fig. 5. The receptivity of the jet is determined through the analysis of spatio-temporal diagrams for different azimuthal modes and different frequencies in the range $0.5<\omega_{f}<14$ at a distance from the nozzle exit of $X=0.6$ diameter. The intensity of the response is then computed as follows. First the axisymmetric trend is calculated according to

$$
A_{0}(t)=\langle A(t, \alpha)\rangle,
$$

where $\langle\cdot\rangle$ designates the angular average, and subtracted from the initial field $A(t, \alpha)$ to yield the azimuthal deformation field $\widetilde{A}(t, \alpha)$ :

$$
\widetilde{A}(t, \alpha)=A(t, \alpha)-A_{0}(t) .
$$

Introducing for any time dependent function $f(t)$ the temporal average $\bar{f}$ and the standard deviation $f=\sqrt{\overline{f^{2}}-\bar{f}^{2}}$, the mean amplitude of the azimuthal field is then estimated through an angular average of all standard deviations according to 


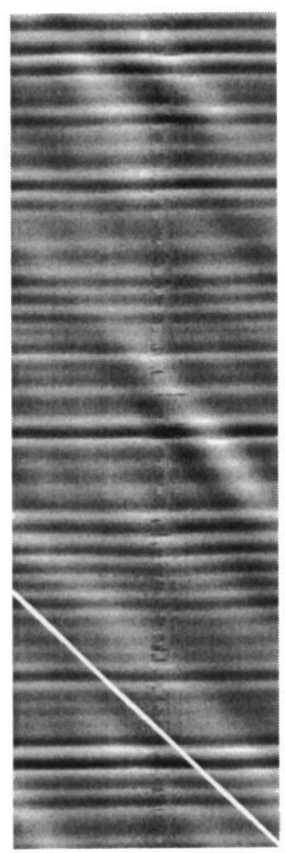

a) $\omega_{\mathrm{f}}=1$

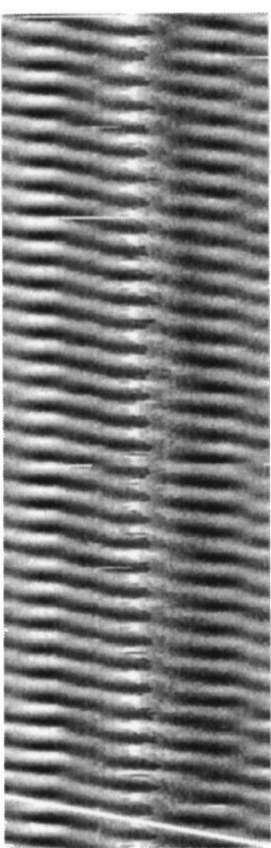

b) $\omega_{\mathrm{f}}=7$

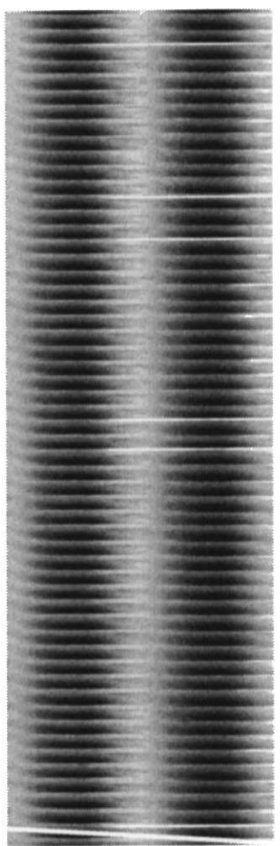

c) $\omega_{\mathrm{f}}=11$

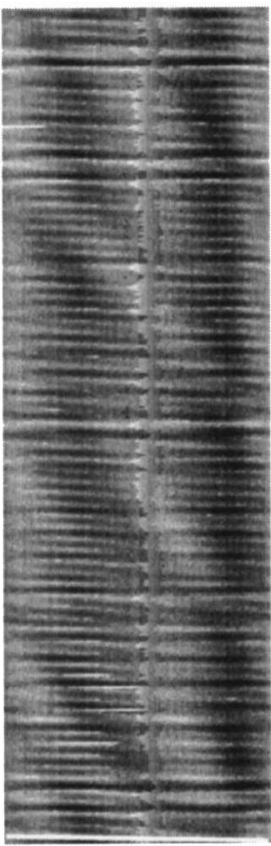

d) $\omega_{\mathrm{f}}=14$
FIG. 8. Spatio-temporal diagrams obtained at 1 diameter of the nozzle exit when $m=+2$ forcing is imposed at various frequencies; $S=0.84$; $\operatorname{Re}=1490$. (a) $\omega_{f}=1$; (b) $\omega_{f}=7$; (c) $\omega_{f}=11 ;(\mathrm{d}) \omega_{f}=14$.

$$
A_{m}\left(v_{\phi}\right)=\left\langle\underline{\left.\tilde{A}\left(t, \alpha+v_{\phi} t\right)\right\rangle},\right.
$$

where $v_{\phi}$ designates the forcing phase velocity. This response amplitude is nondimensionalized with the value in the unforced reference case $A_{2}(0)$.

\section{A. Qualitative results}

\section{1. $m=+2$ forcing}

Let us first compare in Fig. 8 the qualitative response to an $m=+2$ co-rotating helical wave at four forcing frequencies $\omega_{f}=1, \omega_{f}=7, \omega_{f}=11$ and $\omega_{f}=14$. The spatio-temporal diagrams are calculated as previously for a recording of $64 \mathrm{~s}$. In Fig. 8, the contrast has been enhanced on each spatiotemporal diagram separately to ease the identification of structures; the relative amplitude corresponding to the receptivity is discussed later. At the bottom of each diagram, a line is drawn which represents the dimensional phase velocity of the forcing $v_{\phi_{f}}=\omega_{f} / \mathrm{m}$. Some noisy stripes are identified in Fig. 8(d) which result from image processing errors.
The forcing at the natural frequency [see Fig. 8(a)] is surprisingly inefficient at regularizing the $m=+2$ mode. In particular the $m=0$ mode remains present. We have checked further downstream that the development of the double-helix was not enhanced by the inlet forcing at $\omega_{f}=\omega_{\text {nat }}$ despite the optimal tuning of the forcing. When the forcing frequency is increased to $\omega_{f}=7$, the spatio-temporal diagram [see Fig. 8(b)] demonstrates a clear response of the flow at the prescribed frequency, although one can distinguish as a watermark traces of the natural irregular low-frequency wave. Note that, in contrast to Fig. 8(a), the $m=0$ mode, signalled by the horizontal stripes, has disappeared. In one of our experimental runs, the results at $X=1.5$ for $m=+2$ and $\omega_{f}=7$ are exceptionally free from axisymmetric perturbations and this has enabled us to compare the amplitude of the disturbances at $X=1$ and $X=1.5$, as done in Fig. 9. It is found that the amplitude effectively grows in the downstream direction. In Fig. 9, an arrow indicates the sense of rotation in time of the disturbance which is the same as that (a) $\mathrm{m}=+2 ; \mathrm{X}=1$

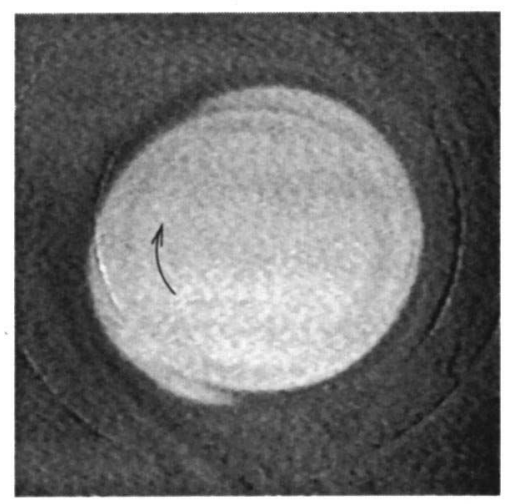

(b) $\mathrm{m}=+2 ; \mathrm{X}=1.5$

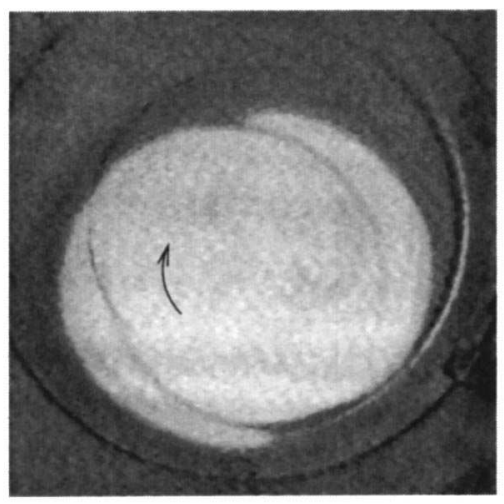

FIG. 9. Cross-cuts obtained at the forcing frequency $\omega_{f}=7$ for $m=+2$ at $X=1$ (a) and $X=1.5$ (b); $S=0.84 ; \operatorname{Re}=1490$. 
(a) $\mathrm{m}=-2$

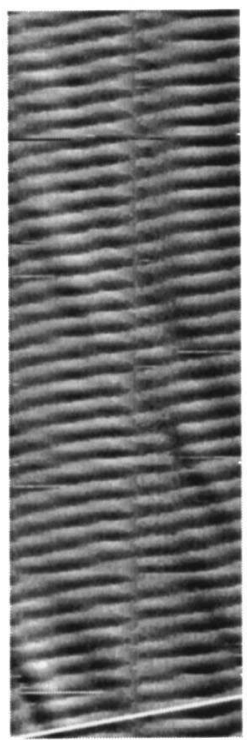

$\omega_{\mathrm{f}}=7$

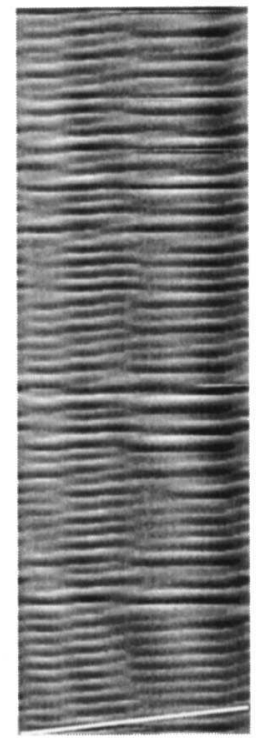

$\omega_{\mathrm{f}}=11$ (b) $m= \pm 3$

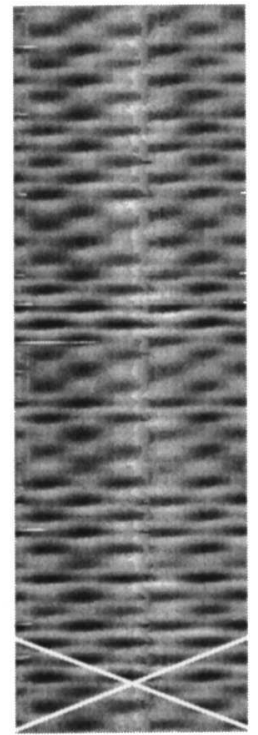

$\omega_{\mathrm{f}}=4.5$

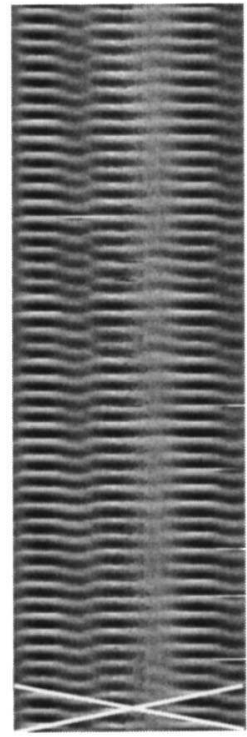

$\omega_{\mathrm{f}}=9$
FIG. 10. Spatio-temporal diagrams obtained at $X=1$ diameter of the nozzle exit when the $m=-2$ (a) and $m= \pm 3$ (b) forcing is imposed at various forcing frequencies; $S=0.84$; $\operatorname{Re}=1490$ of the underlying jet. Note the presence of dye filamentation which breaks the full symmetry of the $m=+2$ perturbation. Considering the rotation of the perturbation around the jet core, the arms of the double helix present dye filamentation at the rear of the perturbation. This is the manifestation of the so-called stripping or erosion process.

The largest response to the forcing is obtained for $\omega_{f}=11$ [see Fig. 8(c)] where the helical $m=+2$ wave is synchronized with the $m=0$ rollers. In this case, the natural frequency of the $m=0$ mode is $\omega_{0}=8$ close to the forcing value, and both waves are strongly affected by the forcing. When the frequency is further increased as for $\omega_{f}=14$ in Fig. 8(d), the low-frequency natural mode $m=+2$ appears again and is irregular, as if no forcing were applied to the flow. By contrast, the axisymmetric $m=0$ mode responds to the forcing.

It should finally be noted that the flow does not show any receptivity to mode $m=-4$, which is automatically forced by the forcing device together with mode $m=+2$.

\section{2. $m=-2$ forcing}

Figure 10(a) presents spatio-temporal diagrams reflecting the response of the flow to $m=-2$ forcing at frequency $\omega_{f}=7$ and $\omega_{f}=11$. In both cases, the qualitative response is signaled by the alignment between the observed structures and the forced phase velocity. Still, the natural $m=+2$ helix is identified as a watermark in Fig. 10(a). Not shown here are the results at lower and higher frequencies where the natural double-helix takes over and no response to the forcing was found.

Figure 4(a), which was introduced in Sec. III in order to describe the construction rules of spatio-temporal diagrams, represents a cross-section at $X=1, \omega_{f}=7$ and $m=-2$ and it is the complementary picture to Fig. 9(a) at the same streamwise location and frequency but for the opposite mode. In Fig. 4(a), the arrow indicates the direction of rotation of the perturbation which is opposite to the background flow. Comparison of both figures suggests that the receptivity of the flow is similar for the co-rotative $m=+2$ and the counterrotative $m=-2$ at $\omega_{f}=7$.

Finally, note that the flow does not show any receptivity to mode $m=+4$, which is automatically forced by the forcing device together with mode $m=-2$.

\section{3. $m= \pm 3$ forcing}

Figure 10(b) represents analog results to those of Fig. 10 (a) for $m= \pm 3$. The checkboard-type spatio-temporal diagrams obtained for $\omega_{f}=4.5$ demonstrate that the jet responds as well to a co-rotative excitation $m=+3$ as to counterrotative excitation $m=-3$.

Figure 11 demonstrates the receptivity of the flow to the forcing further downstream through a comparison of a LIF cross-section at $X=1$ and at $X=6$. The instantaneous Fig. 11(b) displays a threefold symmetry. Three arms branch out of the jet core and roll up into mushroom-type structures. This structure differs from the natural helices. Figure 7(a) indeed demonstrates that the ends of the two arms of the double-helix roll up into single-signed end vortices. This contrasts with the mushroom-type end structures observed in Fig. 11(b), which reveal axial vorticity of both signs. We suggest that the structure could be a consequence of a superposition of $m=+3$ and $m=-3$ modes.

\section{B. Quantitative results}

Quantitative results are displayed in Fig. 12. For each azimuthal wavenumber and each forcing frequency, the response amplitude has been measured on three different spatio-temporal diagrams recorded at different times. The plotted value is the mean of these three values and the error bar their standard deviation. 
(a) $\mathrm{X}=1$

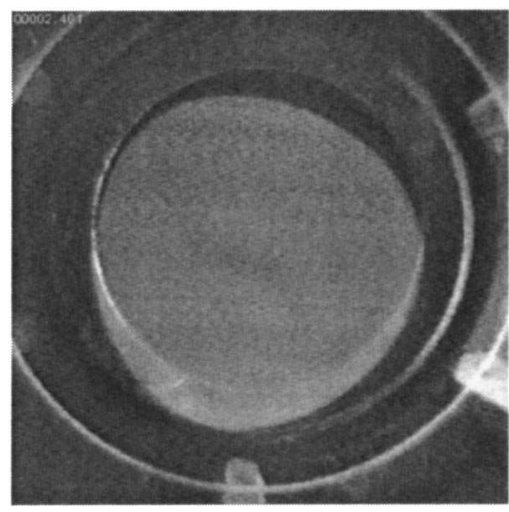

(b) $X=6$

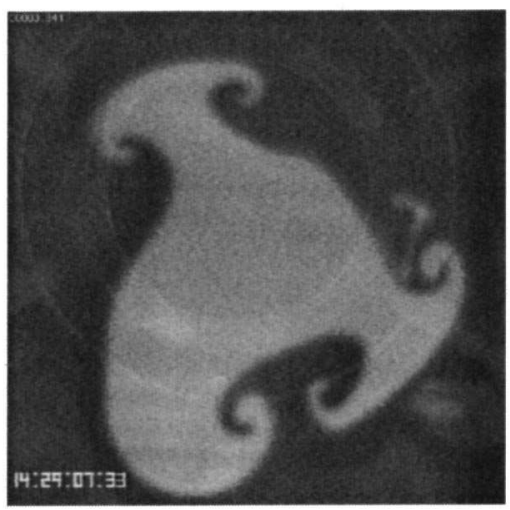

FIG. 11. Cross-cuts obtained at the forcing frequency $\omega_{f}=7$ and $m= \pm 3$ for $X=1$ (a) and $X=6$ (b); $S=0.84 ; \operatorname{Re}=1490$.
A brief summary of the results is that, whatever forcing mode is considered, the response is strongly peaked in the vicinity of $\omega_{f}=8-10$. For a $m=+2$ forcing, we thereby demonstrate that the maximum response is obtained for a frequency one order of magnitude higher than the natural frequency. The swirling jet does not respond to forcing frequencies which are either too low or too high. This result is quite surprising since one would have imagined that the prevailing frequency in the unforced case could easily be triggered through external forcing at this precise frequency setting.

Finally, we have examined the response of the swirling jet to the forcing of different azimuthal modes and frequencies in the breakdown regime $(S \geqslant 1.3)$. The breakdown appears insensitive to these control attempts; it seems to prevent any instability development. Moreover, for $S>1.3$, the stagnation point corresponding to vortex breakdown soon migrates close to the nozzle exit, thereby inhibiting any LIF measurements.

\section{DISCUSSION}

The main findings of the present experimental study are as follows. PIV measurements in the prebreakdown regime have been conducted which confirm the results of Billant et $a .^{7}$ The vorticity fields obtained from finite differencing of the velocity fields in the cross-sectional plane demonstrate the screened nature of the vortex at the nozzle exit. Furthermore, it is seen that vortex breakdown is associated with the appearance of negative vorticity on the jet axis as demonstrated by vorticity plots in the longitudinal plane. Forced experiments at $\operatorname{Re}=1490$ and $S=0.86$ have been conducted. It is shown that the receptivity of the jet is very poor when the forcing is set to the natural prevailing azimuthal mode and frequency. In contrast, a strong response is observed for both co-rotating and counter-rotating forced azimuthal modes $(m= \pm 2, m= \pm 3$ ) for frequencies about one order of magnitude larger than the natural frequency prevailing in the absence of forcing.

A striking result of the study is the extreme robustness of the vortex breakdown, which remains unaffected by azimuthal forcing at the nozzle periphery with the present actuator. This might be understood in at least one of the following manners: according to Squire ${ }^{18}$ or Wang and Rusak, ${ }^{19}$ the mechanism leading to vortex breakdown is a core mechanism related to the Kelvin waves (or inertial waves) living in the core of the vortex. Alternatively, the appearance of negative azimuthal vorticity, claimed by Brown and Lopez ${ }^{12}$ to be responsible for vortex breakdown, also takes place near the swirling jet axis. The present actuator is efficient in manipulating the vorticity sheet separation; it acts at the periphery of the vortex. As one would have guessed from nonrotating jet experiments, this type of actuator is efficient in controlling instabilities that develop at the periphery but not in the core of the vortex. This may be the reason for the failure of the present forcing device in controlling vortex breakdown. (a) $m=+2$

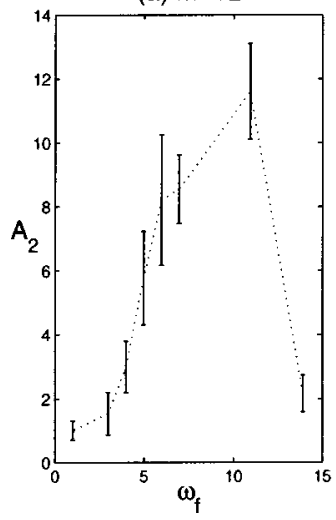

(b) $m=-2$

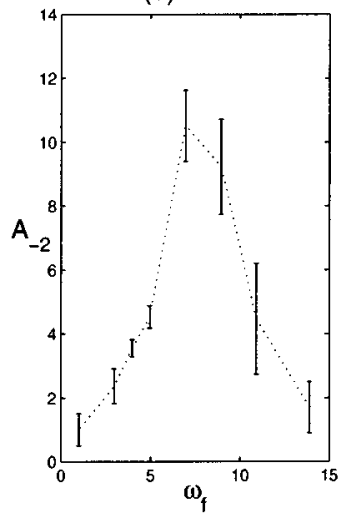

(c) $m= \pm 3$

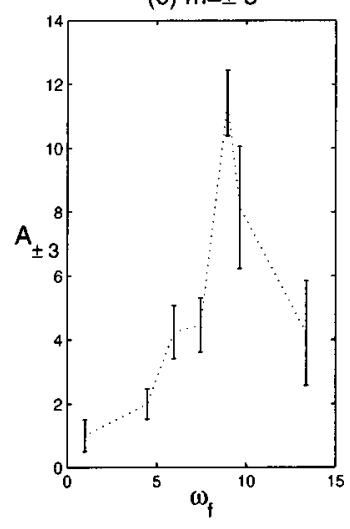

FIG. 12. Response amplitude to the forcing as a function of $\omega_{f}$ at $X=0.6$ and (a) $m=+2$, (b) $m=-2$ and (c) $m= \pm 3 ; S=0.84 ; \mathrm{Re}=1490$. 


\section{ACKNOWLEDGMENTS}

The authors wish to thank A. Garcia for his technical assistance and enthusiasm as well as T. Loiseleux, R. GodoyDiana and L. Tuckerman for helpful suggestions. This work has benefitted from the financial support by the Direction Générale de l'Armement (DGA) under Grant PEA No. 99001004707588, "Eclatement tourbillionnaire."

${ }^{1}$ W. Althaus, C. Brücker, and C. Weimer, "Breakdown of slender vortices," in Fluid Vortices, edited by S. I. Green (Kluwer Academic, Dordrecht, 1996), Chap. 9, pp. 373-426.

${ }^{2}$ J. Delery, "Aspects of vortex breakdown," Prog. Aerosp. Sci. 30, 1 (1994).

${ }^{3}$ A. Mitchell and J. Delery, "Research into vortex breakdown control," Prog. Aerosp. Sci. 37, 385 (2001).

${ }^{4}$ J. Faler and S. Leibovich, "Disrupted states of vortex flow and vortex breakdown," Phys. Fluids 20, 1385 (1977).

${ }^{5}$ T. Loiseleux and J.-M. Chomaz, "Breaking of rotationnal symmetry in a swirling jet experiment," Phys. Fluids 15, 511 (2003).

${ }^{6} \mathrm{~J}$. Panda and D. McLaughlin, "Experiments on the instabilities of a swirling jet," Phys. Fluids 6, 263 (1994).

${ }^{7}$ P. Billant, J.-M. Chomaz, and P. Huerre, "Experimental study of vortex breakdown in swirling jets," J. Fluid Mech. 376, 183 (1998)

${ }^{8}$ E. Hopfinger and G. van Heijst, "Vortices in rotating fluids," Annu. Rev. Fluid Mech. 25, 241 (1993).
${ }^{9}$ T. Loiseleux, Ph.D. thesis, Ecole Polytechnique, 1999.

${ }^{10}$ M. Raffel, C. Willert, and J. Kompenhans, Particle Image Velocimetry: A Practical Guide (Springer, New York, 1998).

${ }^{11} \mathrm{~F}$. Gallaire and J.-M. Chomaz, "Mode selection in swirling jet experiments: a linear stability analysis," J. Fluid Mech. 494, 223 (2003).

${ }^{12}$ G. Brown and J. Lopez, "Axisymmetric vortex breakdown. Part 2: Physical mechanisms," J. Fluid Mech. 221, 553 (1990).

${ }^{13}$ D. Darmofal, "The role of vorticity dynamics in vortex breakdown," AIAA Pap. 93-3036 (1993).

${ }^{14}$ D. Rockwell, M. Ozgoren, and B. Sahin, "Vortex breakdown from a pitching delta wing incident upon a plate: Flow structure as the origin of buffet loading," J. Fluids Struct. 16, 295 (2002).

${ }^{15} \mathrm{X}$. Yin, D. Sun, M. Wei, and J. Wu, "Absolute and convective instability character of slender viscous vortices," Phys. Fluids 12, 1062 (2000).

${ }^{16}$ M. Ruith, P. Chen, E. Meiburg, and T. Maxworthy, "Three-dimensional vortex breakdown in swirling jets and wakes: direct numerical simulation," J. Fluid Mech. 486, 331 (2003).

${ }^{17}$ J. C. Lasheras, A. Lecuona, and P. Rodriguez, "Three-dimensional structure of the vorticity field on the near region of laminar co-flowing forced jets," in The Global Geometry of Turbulence (Plenum, New York, 1991).

${ }^{18}$ H. B. Squire, "Analysis of the vortex breakdown phenomenon," in Miszallaneen der Angewandten Mechanik (Akademie Verlag, Berlin, 1960), p. 306.

${ }^{19} \mathrm{~S}$. Wang and Z. Rusak, "The dynamics of a swirling flow in a pipe and transition to axisymmetric vortex breakdown," J. Fluid Mech. 340, 177 (1997). 